\title{
Adverse reaction regarding titanium-based marker clip: case report of a potential complication
}

This article was published in the following Dove Press journal:

International Medical Case Reports Journal

\section{Urszula Wegner \\ Sarah Rainford}

Department of Breast Imaging, Kettering General Hospital, Kettering, UK
Correspondence: Urszula Wegner Manchester University Hospitals, Nightingale Centre, Southmoor Road, Manchester M23 9QZ, UK

$\mathrm{Tel}+44$ I6I 29I 4444

Email Urszula.Wegner@mft.nhs.uk

\begin{abstract}
Adverse reaction to titanium-based breast marker clip is a very uncommon complication in medicine. We report a 65 -year-old female patient who was referred with left breast lumpiness to the assessment clinic. Breast radiology with interesting sequelae following titanium clip insertion, in combination with literature review, will be discussed. The patient had a history of adverse skin reactions to base metals. However, no allergy specifically to titanium was reported. Imaging findings with regard to the symptomatic breast were nonspecific; thus, multiple core biopsies were taken, and titanium clip was inserted. Subsequent histological examination showed benign changes. However, 7 weeks later, the marked area became very tender and the same patient re-presented to the clinic, requesting removal of the metallic component. Following multidisciplinary meetings and discussions, the person underwent ultrasound-guided vacuum biopsy and the clip was removed. The symptoms completely resolved after the successful procedure, and histological examination revealed lymphocytic infiltration of the tissues. Reactions to titanium-based marker clips are rare and only limited records have been found in the literature. More cases need to be reported in order to establish the full significance of such adverse sequelae. It is recommended that medical history with regard to allergies, prior titanium clip insertions, should be discussed.
\end{abstract}

Keywords: clip marker, breast imaging, sonography, mammography, biopsy

\section{Introduction}

Adverse reaction to metallic marking device, including titanium, is an unlikely scenario following minor breast interventions, namely clip placements.

This may become a potential cause of pain and discomfort with regard to the affected breast.

We report a unique case of a female patient who presented with undesired effects resulting from titanium-based clip insertion. While clip markers are commonly used for better tracking of biopsy sites, such reactions are very uncommon, and hence, adequate diagnosis may be challenging to establish.

Breast imaging, physical examination, and literature review will be analyzed.

\section{Case report}

A 65-year-old woman presented with left breast lumpiness to the symptomatic unit and appointment regarding triple breast assessment clinic was arranged.

Physical examination of the symptomatic left breast revealed no suspicious findings. 
Breast radiology showed a mammographic asymmetry within the upper outer quadrant of the left breast (Figure 1). The level of suspicion for malignancy was low, and this was therefore assigned BI-RADS 4A.

This persisted on additional views. High-resolution sonography revealed no suspicious focal mass. There were only some minimal fibrocystic changes which could potentially correlate with indeterminate mammographic findings. However, the sonographic examination was noncontributory.

The imaging was reviewed and decisions regarding core biopsy with titanium clip placement were made.

Patient informed the team that had some mild adverse skin reactions to base metals previously; namely common materials such as copper, lead, nickel or aluminum used in costumes or jewellery. There was no history of previous medical interventions, autoimmune diseases, and atopic dermatitis, and she was not aware of any allergy or prior contact with titanium.

Since the asymmetry in keeping with an area of interest was well seen on mammography, an open coil-shaped hydro-mark titanium clip was chosen to enable the potential follow-up and exact correlation with sonography. The hydro-mark clips are stainless, based on hydrogel technology, and consist of both hydrogel and titanium. These markers hydrate the site of interest after deployment and subsequently enable long-term visualization with both ultrasound and T2-weighted MRI sequence. Moreover, the leftsided mammographic abnormality regarding our patient was nonpalpable. Thus, the titanium-based clip was used in order to mark the sampled site and provide good correlation across all other breast imaging modalities that could be used for further verification in the future if required.

Post-clip mammogram confirmed that the location of the marker corresponded well with the mammographic abnormality (Figure 1).

Subsequent histopathological examination revealed benign fibrotic changes with no evidence of malignancy or atypia. Thus, given the positive final diagnosis, no further imaging was required, and the patient was discharged.

However, 7 weeks later, the same person re-presented with increasing pain and discomfort within the upper outer quadrant of the left breast.

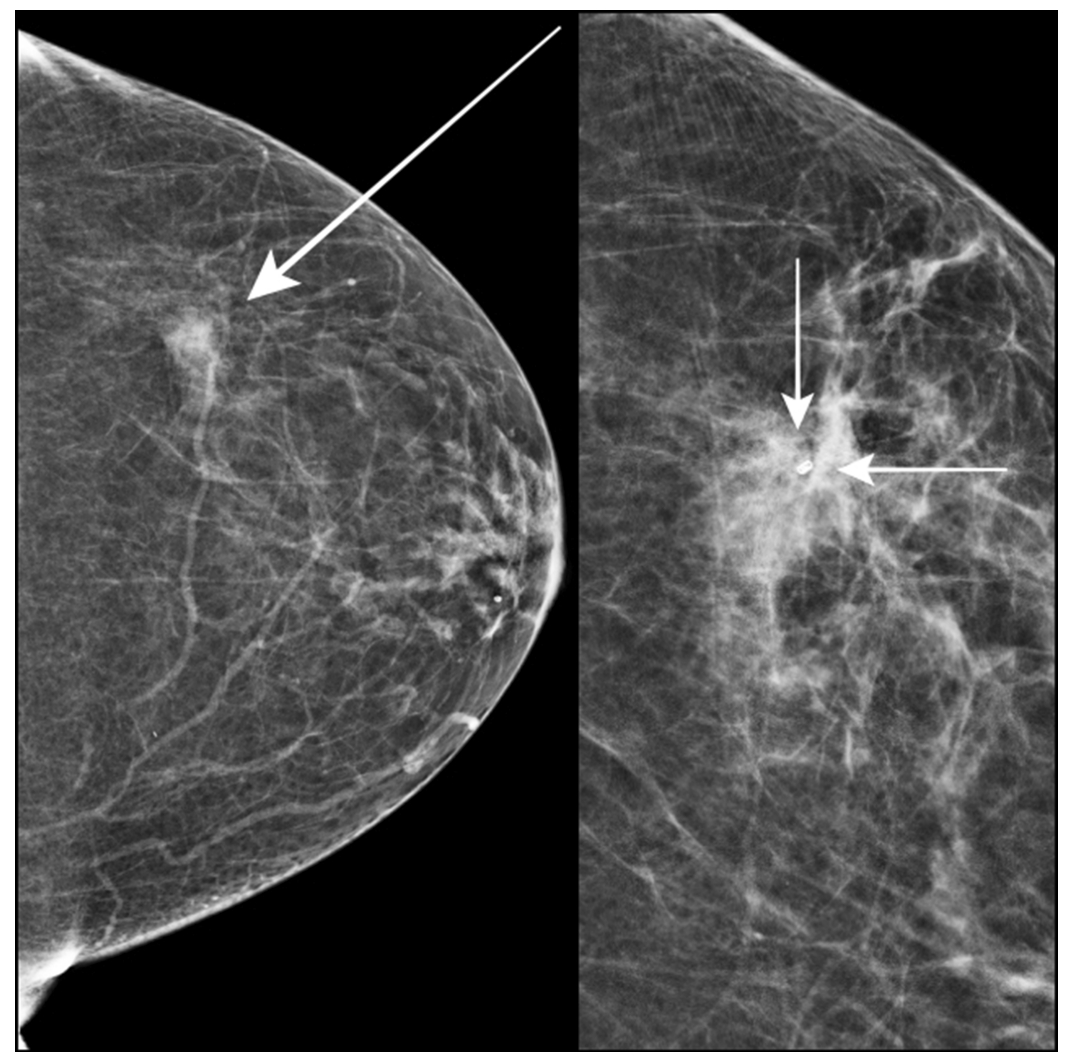

Figure I In 2017, a 65-year-old female patient presented with left breast lumpiness to the symptomatic breast unit. Mammography revealed an indeterminate, low-density area within the upper outer quadrant of the left breast (long arrow). This was subject to core biopsies and subsequent titanium clip placement (short arrows). 
High-resolution ultrasound examination and mammography were again performed. No drainable collection or abscess were visualized (Figure 2). The marker clip was identified, and the location correlated with patient's tenderness (Figure 2). Otherwise, the appearances on imaging were unremarkable.

Therefore, following a multidisciplinary meeting, MRI and second professional opinion were requested. These were noncontributory, demonstrated neither any new significant findings nor suggested to alter the management.

The woman continued to be symptomatic, felt persisting increasing discomfort, itching, and insisted on the removal of the metallic component. There were no associated dermatologic or systemic manifestations. An abscess or other drainable collection post-biopsy were excluded with an ultrasound and suspicion of adverse reaction to titanium was raised.

However, the patient was informed that it is still uncertain if any invasive interventions would fully treat her symptoms.

Possible options regarding surgical and radiological interventional procedures were discussed.

Following conversations and permission granted in full knowledge of risks and benefits, the patient decided to undergo ultrasound-guided vacuum clip excision. The procedure was successfully performed under local anesthesia. The titanium clip within the tissue sample was identified (Figure 3).

No immediate or delayed complications were observed. Moreover, the undesired symptoms subsequently resolved.

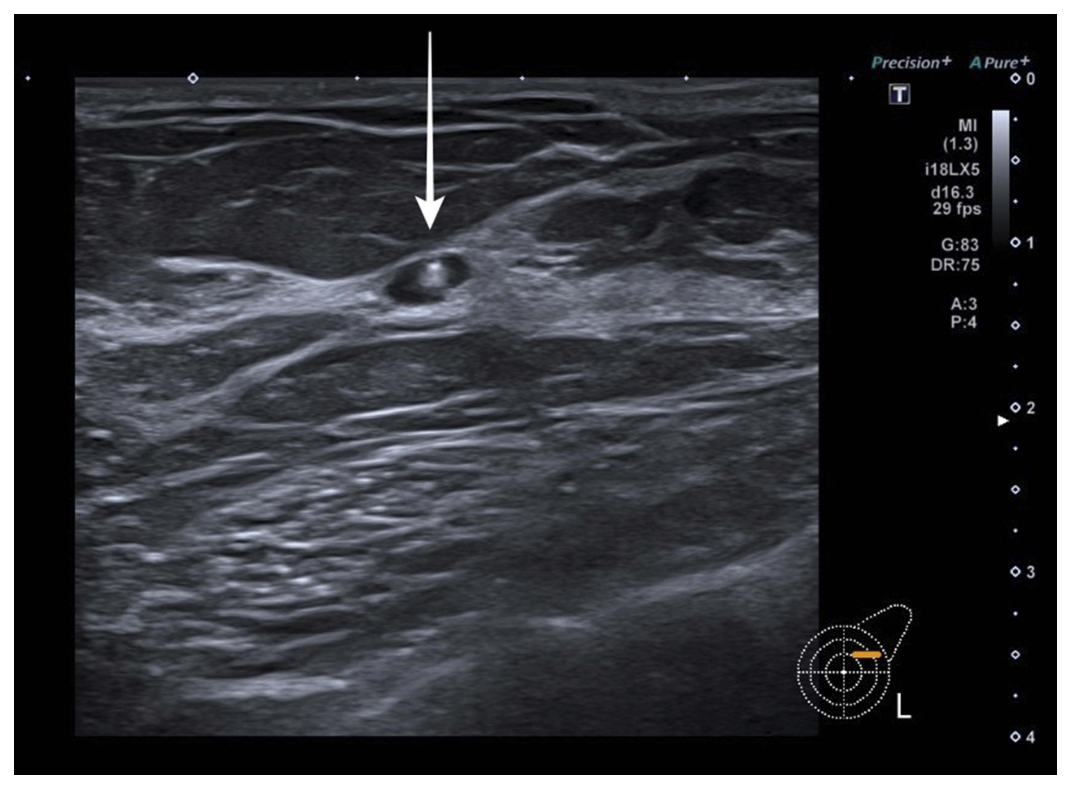

Figure 2 Seven weeks later, the same patient re-presented to the breast unit with a history of increasing pain and discomfort with regard to the marked upper outer left breast. High-resolution ultrasound revealed unremarkable titanium clip marker (arrow). There was no drainable collection or any significant findings to justify the symptoms. Location of the pain correlated well with the patient's tenderness.

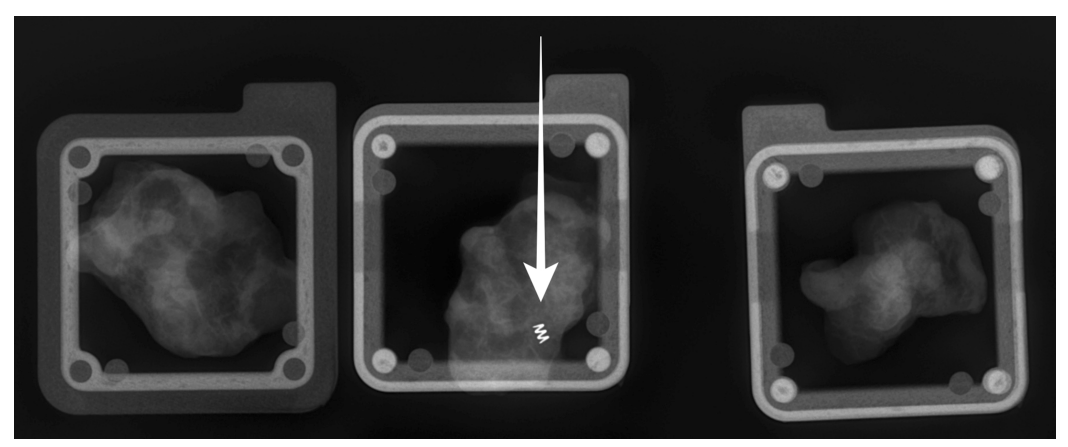

Figure 3 The same symptomatic patient requested removal of the metallic component and a vacuum clip excision was made. The procedure was successful, and titanium clip within the biopsy specimens was visualized (arrow). 


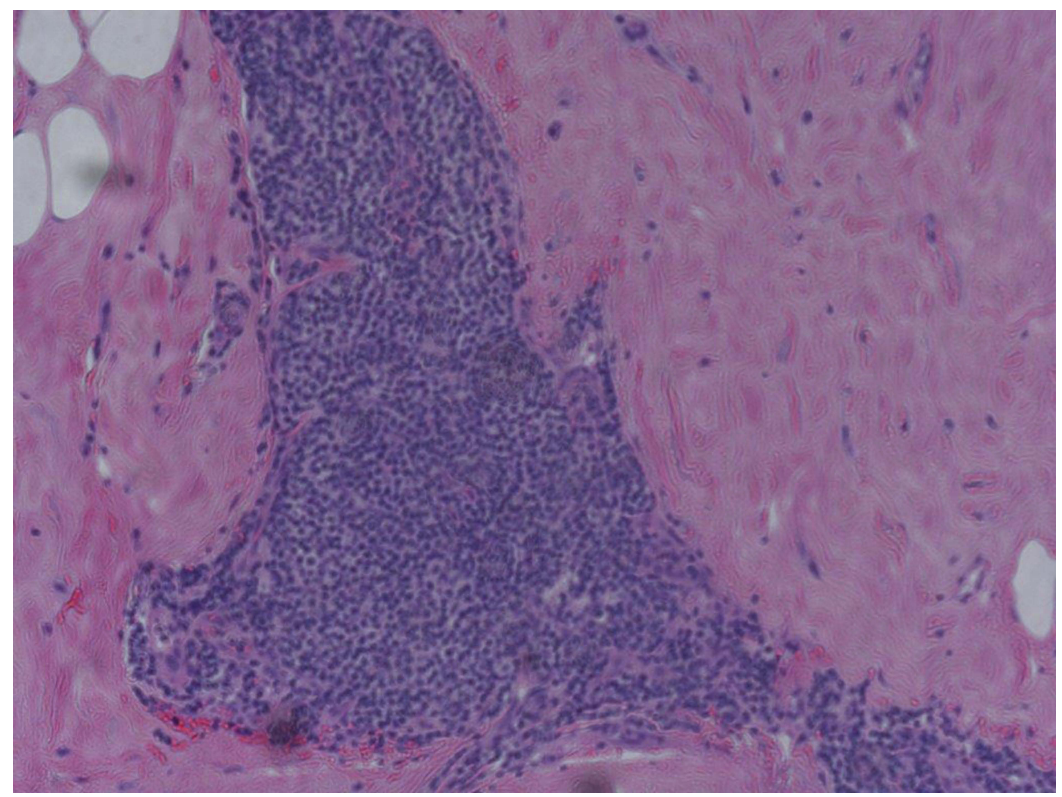

Figure 4 Microphotograph illustrates histopathology of the same patient as in Figures I-3.

The total duration of the titanium clip implantation within the affected breast was 15 weeks. Histopathological examination demonstrated lymphocytic infiltration of the tissues (Figure 4). No overt foreign body granuloma was identified. Histology in combination with clinical history and breast radiology were suggestive of a delayed reaction and sensitization to titanium material.

\section{Discussion}

The presented case stimulated an ongoing point of discussion and raised questions with regard to adverse reactions to titanium-based materials.

Due to the rarity of such a phenomenon, it may be challenging to diagnose the potential allergy or any related complications. ${ }^{1}$

Limited cases of titanium sensitivity have been documented in the literature and the clinical presentations were variable. ${ }^{1}$ Some patients presented with pain as in our case. Moreover, there were manifestations with regard to contact dermatitis, severe itching, skin rash, inflammatory granulomatous reactions, or even failure of the orthopedic hardware. $^{1-4}$

An interesting case of previously reported allergic reaction and exacerbation of atopic dermatitis following three titanium markers insertion in a female undergoing breast conserving surgery demonstrated similar time frame regarding the adverse reaction, as in our patient. ${ }^{3}$
Both women presented with delayed reactions approximately 2 months following implantation of the titanium markers. ${ }^{3}$ However, the clinical manifestations were different. Our patient experienced increasing pain and pruritus limited only to the site of metal insertion and there was no associated dermatological disease, whereas the other reported patient exhibited severe exacerbation of widespread atopic dermatitis. ${ }^{3}$

The symptoms associated with atopic dermatitis have improved but not fully resolved after the removal of the clip. ${ }^{3}$ In comparison, a full resolution of the symptoms following ultrasound vacuum excision was noted in our patient's case.

Cases of adverse skin reactions and discomfort associated with skin-penetrating bone-anchored conduction implants used to treat patients with conductive hearing loss have also been highlighted. ${ }^{5}$

Even though titanium is widely utilized and considered safe in the medical field, thorough medical history regarding any immune responses or hypersensitivity reactions prior to any device placement should be discussed. ${ }^{3,6}$

However, it is important to highlight the fact that common dedicated allergy skin-patch tests are of limited use, namely, carry the risk of false reactions which may not reflect the adequate immune response. ${ }^{4,6}$

For example, in 2006, a case of hypersensitivity to titanium osteosynthesis in a patient with negative skinpatch test results has been reported and it was necessary 
to remove the offending material in order to achieve the fracture healing. ${ }^{6}$

Thus, it could be equivocal and challenging to establish the underlying cause of the symptoms based on skin-patch testing alone. Lymphocyte transformation test proved to be more sensitive in order to evaluate the suspected titanium allergy in the reported case. ${ }^{6}$

Currently, there are no precise or standard methods to test the potential predispositions to titanium allergy. Thus, further investigations with regard to titanium-associated diagnostic allergy tests would be helpful to establish the full significance of potential sensitization to this material. ${ }^{7}$

Microscopic verification of the biopsy specimens showed lymphocytic infiltration of the tissue suggestive of a possible delayed hypersensitivity reaction. No overt foreign body granuloma was identified.

\section{Conclusion}

Reactions to titanium-based devices are very rare, and to the best of our knowledge, only a few reports have been published in the literature. Thus, such cases may cause a significant diagnostic dilemma and mislead various professionals regardless of experience.

The risk of reaction to titanium is exceptionally uncommon, and more research with regard to potential adverse sequelae associated with these biopsy markers needs to be done.

Full resolution of the symptoms after removal of the offending material can be reassuring and can help establish the diagnosis in the presence of adequate clinical scenario.

Adverse reaction to titanium markers, although rare, could be a possible cause of persistent pain weeks later after insertion of these clips. Further radiological verification and possibly removal of the clips with histopathological examination are required for management of the patient's condition.

\section{Acknowledgment}

We would like to acknowledge a reputable pathologist Dr. Ashraf Ibrahim and thank him for his microphotograph.

\section{Declaration of patient consent}

The authors confirm that written informed consent form has been provided by the patient to have the case details published.

\section{Disclosure}

The authors report no conflicts of interest in this work.

\section{References}

1. Wood MM, Warshaw EM. Hypersensitivity reactions to titanium diagnosis and management. Dermatitis. 2015;26(1):7-25. doi:10.1097/ DER.0000000000000091

2. Motton S, Gardinal I, Soule-Tholy M, et al. Hurt eczematiforme column (chronicle) of the breast after implementation of a surgical clip. J Gynecol Obstet Biol Reprod (Paris). 2011;40(2):174-177. doi:10.1016/j.jgyn.2010.07.010

3. Tamai K, Mitsumori M, Fujishiro S, et al. A case of allergic reaction to surgical metal clips inserted for postoperative boost irradiation in a patient undergoing breast-conserving therapy. Breast Cancer. 2001;8 (1):90-92.

4. Yamauchi R, Morita A, Tsuji T. Pacemaker dermatitis from titanium. Contact Derm. 2000;42:52.

5. Holgers KM, Roupe G, Tjellstrom A, Bjursten LM. Clinical, immunological and bacteriological evaluation of adverse reactions to skinpenetrating titanium implants in the head and neck region. Contact Dermatitis. 1992;27(1):1-7. doi:10.1111/j.1600-0536.1992.tb05189.x

6. Peter T, Wolf-Dieter B, Sonja M, et al. Hypersensitivity to titanium osteosynthesis with impaired fracture healing, eczema, and T-cell hyperresponsiveness in vitro: case report and review of the literature. Contact Dermatitis. 2006;55(4):199-202. doi:10.1111/j.1600-0536.20 06.00931.x

7. Goutam M, Giriyapura C, Mishra SK. Titanium allergy: a literature review. Indian J Dermatol. 2014;59(6):630. doi:10.4103/0019-5154. 143526

\section{Publish your work in this journal}

The International Medical Case Reports Journal is an international, peer-reviewed open-access journal publishing original case reports from all medical specialties. Previously unpublished medical posters are also accepted relating to any area of clinical or preclinical science. Submissions should not normally exceed 2,000 words or 4 published pages including figures, diagrams and references. The manuscript management system is completely online and includes a very quick and fair peer-review system, which is all easy to use. Visit http://www.dovepress.com/testimonials.php to read real quotes from published authors. 